\title{
Example of a Poincaré anomaly in relativistic quantum mechanics*
}

\author{
Stefan Lenz \\ Center for Theoretical Physics, Sloane Physics Laboratory \\ Yale University, New Haven, CT 06520-8120, U.S.A. \\ Bernd Schreiber \\ Massachusetts Institute of Technology \\ Center for Theoretical Physics, Cambridge, MA 02139, U.S.A.
}

(November 19, 2018)

\begin{abstract}
The Poincaré algebra of classical electrodynamics in one spatial dimension is studied using light-cone coordinates and ordinary Minkowski coordinates. We show that it is possible to quantize the theory by a canonical quantization procedure in a Poincaré invariant manner on the light-cone. We also show that this is not possible when using ordinary coordinates. The physical reason of this anomaly is analysed.

PACS number: 03.65.Pm, 11.10.Ek, 11.10.Kk
\end{abstract}

Typeset using REVTEX

\footnotetext{
*This work is supported in part by funds provided by the U. S. Department of Energy (D.O.E.) under cooperative research agreement DE-FC02-94ER40818 (B.S.) and DE-FG02-91ER40608 (S.L.) and by a Feodor-Lynen-Fellowship of the Alexander von Humboldt-Foundation (B.S.).
} 


\section{INTRODUCTION}

It is well known that it is generally not possible to formulate relativistic quantum mechanics with a finite number of degrees of freedom, due to the existence of negative energy solutions of the relativistic wave equations. These solutions are interpreted as antiparticles. Wave packets with an extension smaller than the particle's Compton wavelength $\lambda_{c}=1 / m$, contain considerable admixtures of these antiparticle solutions (see e.g. [1]). Therefore a many body description of relativistic quantum systems is usually inevitable.

We will show, however, that it is possible to quantize gauge theories in one space and one time dimension (1+1-dimensions) on a truncated Fock space, while preserving Lorentz invariance, if one uses light-cone coordinates. More specifically, in this paper we will analyse the difference between light-cone quantization and quantization in ordinary coordinates in a Hamiltonian formulation of electrodynamics in 1+1-dimensions. We will explicitly show that canonical quantization of this theory is possible in a Poincaré invariant manner in lightcone coordinates but not in ordinary coordinates. This result means that one encounters a quantum mechanical anomaly when using an equal-time quantization procedure. Position space matrix elements for the anomalous contribution to the Poincaré algebra can be inter-

preted in terms of particle trajectories which make an intuitive interpretation of the results possible.

In a pioneering paper, Dirac formulated the basic requirements necessary for combining the Hamiltonian formulation of dynamics with special relativity [2]. The principle of relativity demands invariance of physical laws under continuous coordinate transformations, which are boosts and translations

$$
x^{\mu^{\prime}}=\Lambda_{\nu}^{\mu} x^{\nu}+a^{\mu} \quad \Lambda_{\mu}^{\nu} \Lambda^{\mu \rho}=g^{\nu \rho} .
$$

\footnotetext{
1 Throughout this paper we set $\hbar=c=1$.

${ }^{2}$ In $3+1$ dimensions also invariance under rotations has to be demanded.
} 
This so called Poincaré group is determined by the Lie algebra of its generators

$$
\{H, P\}=0, \quad\{K, P\}=H, \quad\{K, H\}=P .
$$

$H:=P^{0}$ is the Hamiltonian, $P:=P^{1}$ is the momentum and $K$ the boost generator of the physical system under consideration. $\{A, B\}$ is the standard Poisson bracket [3], with $\left\{x_{n}, p_{m}\right\}=\delta_{n m} \cdot x_{m}$ are the positions of the particles and $p_{m}$ the corresponding canonical momenta. Note that although a Hamiltonian formulation is not manifestly covariant, the theory is still Lorentz invariant if the Poincaré algebra is closed. $K$ generates a canonical transformation of the phase space variables $\left(x_{m}, p_{m}\right)$ which connects different reference frames. Due to the last two brackets in (2), $P^{\mu}=\left(P^{0}, P^{1}\right)$ transforms like a Lorentz vector. The first bracket expresses translation invariance.

Quantum mechanically, one has to replace the Poisson brackets by $-i[$,$] where [$,$] is$ the commutator. The quantum Poincaré algebra therefore is

$$
[\hat{H}, \hat{P}]=0, \quad[\hat{K}, \hat{P}]=i \hat{H}, \quad[\hat{K}, \hat{H}]=i \hat{P}
$$

A closed quantum Poincaré algebra means that a unitary transformation

$$
\hat{U}(\lambda):=\exp (i \lambda \hat{K})
$$

exists which connects quantities in different Lorentz frames, where $\hat{K}$ is the generator of this unitary transformation. The existence of this unitary transformation is a necessary and sufficient condition for a Lorentz invariant formulation of quantum mechanics. In general the existence of a canonical transformation does not guarantee the existence of the corresponding unitary transformation. One example for this is the axial anomaly in QED where the classical theory is invariant under chiral rotations but the quantized theory is not [5] 7 .

A Hamiltonian formulation of a dynamical system using ordinary Minkowski coordinates $x^{0}, x^{1}$ corresponds to specifying the initial conditions on a space-like hypersurface $x^{0}=$ const in Minkowski space. Another possibility is offered by the use of light-cone coordinates

$$
x^{+}=\frac{x^{0}+x^{1}}{\sqrt{2}} \quad x^{-}=\frac{x^{0}-x^{1}}{\sqrt{2}}
$$


where a new time variable $x^{+}$and a new space variable $x^{-}$are introduced [2]. Initial conditions are now specified on a lightlike hypersurface $x^{+}=$const. As with the ordinary coordinates, the generators of the Poincaré group are the components of the momentum vector:

$$
H_{L C}:=P_{+}=\frac{P_{0}+P_{1}}{\sqrt{2}} \quad P_{L C}:=P_{-}=\frac{P_{0}-P_{1}}{\sqrt{2}}
$$

and a boost generator $K_{L C}$. The quantum Poincaré algebra has the form

$$
\left[\hat{H}_{L C}, \hat{P}_{L C}\right]=0 \quad\left[\hat{K}_{L C}, \hat{H}_{L C}\right]=-i \hat{H}_{L C} \quad\left[\hat{K}_{L C}, \hat{P}_{L C}\right]=i \hat{P}_{L C}
$$

These relations are found by using the definitions of $P_{+}$and $P_{-}$(5) and the Heisenberg brackets (3). Quantum mechanically, the choice of one of the two formulations does not necessarily lead to the same theory. This will be demonstrated for classical electrodynamics in one space and one time dimension below.

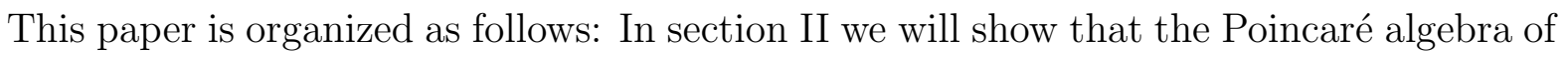
classical electrodynamics closes in 1+1-dimensions. The quantum Poincaré algebra, however, does not close when one is using ordinary coordinates. The physical reason for this anomaly will be explained in terms of retardation effects. Analogous calculations using light-cone coordinates show that no anomaly occurs in this case (sec. III). In section [V] we show that the anomalous contribution to the Poincaré algebra vanishes in the infinite momentum frame. We conclude in section $\square$.

\section{POINCARÉ INVARIANCE FOR ORDINARY COORDINATES}

In one space and one time dimension (1+1 dimensions) the field strength tensor contains only one independent component, and the electric field can easily be expressed by the coordinates of the charged particles [8]. In this paper, we restrict ourselves to 2 particles of equal mass $m$ with charges $e$ and $-e$. The generalization to many particles is straightforward. One obtains the following classical Hamilton function 


$$
H=\sum_{i=1}^{2} \sqrt{p_{i}^{2}+m^{2}}+e^{2}\left|x_{1}-x_{2}\right| .
$$

The remaining two generators of the Poincaré algebra are

$$
\begin{aligned}
& P=\sum_{i=1}^{2} p_{i} \\
& K=\frac{1}{2} \sum_{i=1}^{2}\left(x_{i} \sqrt{p_{i}^{2}+m^{2}}+\sqrt{p_{i}^{2}+m^{2}} x_{i}\right)+\frac{1}{2} e^{2} \epsilon\left(x_{1}-x_{2}\right)\left(x_{1}^{2}-x_{2}^{2}\right)
\end{aligned}
$$

$(\epsilon(x)$ is 1 for positive and -1 for negative argument). It is a simple exercise to show that the Poincaré algebra (2) is closed. The ordering of the first two terms in $K$ is of no significance in the classical theory, but is needed in order to ensure hermiticity of $K$ after quantization.

The reason why it is possible to formulate electrodynamics in one space dimension in a Lorentz invariant way using an instantanous interaction is closely related to the properties of the linear potential, as the interparticle force is constant. For classical particles, such a constant force yields the same dynamics as a retarded force [4], which can be seen in the following way: The origin of retardation effects is that no signal can propagate faster than with the speed of light. Therefore, the force which acts on a particle at position $x_{M}$ (see figure (1), because of a particle travelling on the Minkowski worldline $x_{T}(t)$, is determined by the intersection point $x_{S}$ of the worldline $x_{T}$ with the backward light cone of $x_{M}$. The size and direction of the force only depends on whether the intersection point $x_{S}$ is right or left of $x_{M}$. Because the particle cannot move faster than with the speed of light, the intersection point of the wordline $x_{T}$ with the $t=0$ axis (which determines the instantaneous force) is on the same side as $x_{S}$ with respect to $x_{M}$. Therefore, retardation effects are not important for the specific system considered here.

For our discussion of the quantum Poincaré algebra, we replace the canonical variables $x_{n}$ and $p_{n}$ by the corresponding operators $\hat{x}_{n}$ and $\hat{p}_{n}$. One finds that the first two equations in (3) are fullfilled, but that

$$
[\hat{K}, \hat{H}]=i \hat{P}+\hat{Z}
$$

with a nonvanishing operator 


$$
\begin{aligned}
\hat{Z} & :=\frac{1}{2} e^{2} \sum_{i=1}^{2}\left\{\hat{\Omega}_{i}\left(\hat{x}_{1}^{2}-\hat{x}_{2}^{2}\right)-\left(\hat{\Omega}_{i} \hat{x}_{i}+\hat{x}_{i} \hat{\Omega}_{i}\right)\left(\hat{x}_{1}-\hat{x}_{2}\right)\right\} \\
\hat{\Omega}_{i} & :=\left[\epsilon\left(\hat{x}_{1}-\hat{x}_{2}\right), \sqrt{\hat{p}_{i}^{2}+m^{2}}\right] .
\end{aligned}
$$

This means that the Poincaré invariance of the classical system is destroyed by quantization and that we encounter an anomaly. The anomaly is characterized by the operator $\hat{Z}$.

We will now study matrix elements of this operator in order to obtain an intuitive understanding of the nature of the anomaly. In appendix A, it is proven that

$$
\begin{array}{r}
\left\langle x_{1}^{\prime} x_{2}^{\prime}|\hat{Z}| x_{1} x_{2}\right\rangle=\frac{e^{2} m}{2 \pi}\left\{\epsilon\left(x_{1}^{\prime}-x_{2}^{\prime}\right)-\epsilon\left(x_{1}-x_{2}\right)\right\}\left(x_{1}-x_{2}\right)\left(x_{1}^{\prime}-x_{2}^{\prime}\right) \times \\
\left\{\delta\left(x_{2}-x_{2}^{\prime}\right) \frac{K_{1}\left(m\left|x_{1}-x_{1}^{\prime}\right|\right)}{\left|x_{1}-x_{1}^{\prime}\right|}-\delta\left(x_{1}-x_{1}^{\prime}\right) \frac{K_{1}\left(m\left|x_{2}-x_{2}^{\prime}\right|\right)}{\left|x_{2}-x_{2}^{\prime}\right|}\right\},
\end{array}
$$

where $K_{1}$ is a modified bessel function [9]. For the case that the two particles do not cross each other, $\left(x_{1}-x_{2}\right)$ and $\left(x_{1}^{\prime}-x_{2}^{\prime}\right)$ have the same sign and the matrix element vanishes. Therefore, the first conclusion at this point is that crossing of the particles must be an essential feature for the appearance of the anomaly. We can see this clearly by calculating matrix elements of the first relativistic contribution? in (11)

$$
\hat{Z} \approx \frac{e^{2} i}{2 m^{3}}\left\{\delta\left(\hat{x}_{1}-\hat{x}_{2}\right)\left(\hat{p}_{1}+\hat{p}_{2}\right)\right\}
$$

with eigenfunctions of the nonrelativistic Hamiltonian

$$
\hat{H}=\sum_{i=1}^{2} \frac{\hat{p}_{i}^{2}}{2 m}+e^{2}\left|\hat{x}_{1}-\hat{x}_{2}\right| .
$$

The eigenstates are Airy-functions with the eigenvalues

$$
E_{n}=\left|a_{n}^{\prime}\right|\left(\frac{e^{4}}{m}\right)^{\frac{1}{3}}
$$

where $a_{n}^{\prime}$ are the zeros of the first derivative of the Airy-function (see appendix B). It is easy to show that $\hat{Z}$ of eq.(13) has the following expectation value

\footnotetext{
${ }^{3}$ In the nonrelativistic limit $\sqrt{p^{2}+m^{2}} \approx m+p^{2} /(2 m)$ the Poincaré algebra closes. In this case $K$ is the generator of Galilei transformations.
} 


$$
\left\langle\Psi_{n}|\hat{Z}| \Psi_{n}\right\rangle=\frac{i e^{4}}{4 m^{3}} \frac{1}{E_{n}}
$$

Classically, the particles move periodically with period $T$. Because of the virial theorem, $T \sim \sqrt{E}$, since the potential is linear. Therefore eq.(16) can be written alternatively

$$
\left\langle\Psi_{n}|\hat{Z}| \Psi_{n}\right\rangle \sim \frac{1}{T^{2}}
$$

This result can be explained in the following way: we already pointed out that crossing of the particles is the physical reason for the anomaly. Therefore we expect that the matrix element under consideration is proportional to the number of crossings in a given time interval, i.e. $\sim 1 / T$. It has also to be proportional to the typical time the particles' distance is less than one Compton wavelength. This typical time is proportional to the inverse velocity of the particles when they cross each other which is proportional to the inverse oscillation time $1 / T$. Therefore we find that $\langle Z\rangle \sim 1 / T^{2}$ in agreement with the calculation.

The physical reason for the violation of the Poincaré algebra is related to the fact that quantum mechanically a particle can move faster than the speed of light over distances comparable with its Compton wavelength. This one can see by calculating the free retarded propagator of a relativistic particle in 1+1-dimensions

$$
G_{0}\left(x^{\prime}, t^{\prime} ; x, t\right)=\theta\left(t^{\prime}-t\right) \frac{m\left(t^{\prime}-t\right)}{\pi \sqrt{\left(x^{\prime}-x\right)^{2}-\left(t^{\prime}-t\right)^{2}}} K_{1}\left(m \sqrt{\left(x^{\prime}-x\right)^{2}-\left(t^{\prime}-t\right)^{2}}\right)
$$

$(\theta(x)$ is 1 for positive and 0 for negative argument). Propagation into the spacelike region $\left(x^{\prime}-x\right)^{2}>\left(t^{\prime}-t\right)^{2}$ is possible although it is exponentially suppressed as $K_{1}(m z) \sim \exp (-m z)$ for large arguments. Therefore, retardation effects are important if the particles cross each other. In fig.2 there are two intersection points of the particle worldline with the backward light cone of $x_{M}$ on the left side of $x_{M}$ and one on the right side. The instantaneous force in the Hamiltonian formalism does not describe the dynamics properly because the intersection point of the particle wordline with the $t=0$-axis is on the right side of $x_{M}$, causing a negative force. The retarded force, however, is positive.

If a particle propagates forward in time into a spacelike region in one Lorentz frame then there is another Lorentz frame where this particle propagates backward in time. This 
is interpreted as the presence of an antiparticle. In order to keep Lorentz invariance one is forced to allow for particle-antiparticle production which is not inherent in the quantum mechanical Hamiltonian considered here.

It is possible to get an approximately closed Poincaré algebra, however, including the first relativistic correction. To this end we add to the Hamiltonian an additional potential $h \delta\left(\hat{x}_{1}-\hat{x}_{2}\right)$ which is only nonzero when the particles cross each other. $h$ has to be determined in such a way that the Poincare algebra is closed up to this order. We therefore have for the Hamiltonian and the boost generator

$$
\hat{H}^{\prime}=\hat{H}+h \delta\left(\hat{x}_{1}-\hat{x}_{2}\right), \quad \hat{K}^{\prime}=\hat{K}+\frac{h}{2}\left(\hat{x}_{1}+\hat{x}_{2}\right) \delta\left(\hat{x}_{1}-\hat{x}_{2}\right) .
$$

One finds that the first two equations of (3) still hold and that

$$
\left[\hat{K}^{\prime}, \hat{H}^{\prime}\right]=i \hat{P}+\hat{Z}+\hat{Z}^{\prime}
$$

where $\hat{Z}$ is defined as in eq.(11) and

$\hat{Z}^{\prime}=\frac{h i}{2}\left\{\left(\frac{\hat{p}_{1}}{\sqrt{\hat{p}_{1}^{2}+m^{2}}}+\frac{\hat{p}_{2}}{\sqrt{\hat{p}_{2}^{2}+m^{2}}}\right) \delta\left(\hat{x}_{1}-\hat{x}_{2}\right)+\delta\left(\hat{x}_{1}-\hat{x}_{2}\right)\left(\frac{\hat{p}_{1}}{\sqrt{\hat{p}_{1}^{2}+m^{2}}}+\frac{\hat{p}_{2}}{\sqrt{\hat{p}_{2}^{2}+m^{2}}}\right)\right\}$.

With $\sqrt{\hat{p}_{1}^{2}+m^{2}} \approx m$ and $\sqrt{\hat{p}_{2}^{2}+m^{2}} \approx m$ we have

$$
\hat{Z}^{\prime} \approx \frac{h i}{m}\left[\left(\hat{p}_{1}+\hat{p}_{2}\right) \delta\left(\hat{x}_{1}-\hat{x}_{2}\right)\right] .
$$

Comparing that with $\hat{Z}$ in eq.(13) we find that the Poincaré algebra is closed including the first relativistic correction, if we set

$$
h=-\frac{e^{2}}{2 m^{2}} .
$$

One obtains an intuitive understanding for this additional attractive $\delta$-potential by keeping in mind that it is only non-vanishing when the particles cross each other and it enhances the relative velocity during the crossing. This is in agreement with the qualitative explanation of the anomaly given above.

Higher order relativistic corrections contain derivatives of the $\delta$-function at the origin. Therefore locality of the interaction is lost. 


\section{POINCARÉ INVARIANCE IN LIGHT-CONE COORDINATES}

In the quantized theory one has the following generators of the Poincaré group

$$
\begin{gathered}
\hat{H}_{L C}=\sum_{i=1}^{2} \frac{m^{2}}{2 \hat{p}_{i}}+e^{2}\left|\hat{x}_{1}-\hat{x}_{2}\right| \\
\hat{P}_{L C}=\sum_{i=1}^{2} \hat{p}_{i} \quad \hat{K}_{L C}=\frac{1}{2} \sum_{i=1}^{2}\left(\hat{p}_{i} \hat{x}_{i}+\hat{x}_{i} \hat{p}_{i}\right) .
\end{gathered}
$$

Note that $\hat{K}$ is independent of the interaction and therefore a non-dynamical generator.

It is easy to check that the Poincaré algebra (6) still closes in the quantum theory. This result clearly signals that the quantum mechanical system under consideration must be different from that in ordinary coordinates where Poincaré invariance was violated by quantization. The reason for this is rooted in the structural simplicity of proper Lorentz transformations on the light-cone. The Lorentz tensor reads

$$
\Lambda_{\nu}^{\mu}=\left(\begin{array}{cc}
\exp (\alpha) & 0 \\
0 & \exp (-\alpha)
\end{array}\right),
$$

where $\alpha$ is the velocity of the moving frame. As a consequence, Lorentz transformations are simply scale transformations of the coordinates $x^{+}$and $x^{-}$without mixing them in contrast to ordinary coordinates. Therefore, a particle which moves forward in time in one Lorentz frame will move forward in time in any other Lorentz frame independent of the dynamics under consideration. This fact is reflected in the simple form of the boost generator in (25).

To remove negative energy states (for which one does not have a proper interpretation in a quantum mechanical theory), one can introduce a projection operator on positive momentum states

$$
\hat{Q}:=\int_{0}^{\infty} d p|p\rangle\langle p|
$$

It is easy to check that the projected operators

$$
\hat{H}_{Q}:=\hat{Q} \hat{H}_{L C} \hat{Q} ; \quad \hat{P}_{Q}:=\hat{Q} \hat{P}_{L C} \hat{Q} ; \quad \hat{K}_{Q}:=\hat{Q} \hat{K}_{L C} \hat{Q}
$$


still fulfill a closed Poincaré algebra

$$
\left[\hat{H}_{\hat{Q}}, \hat{P}_{\hat{Q}}\right]=0, \quad\left[\hat{K}_{\hat{Q}}, \hat{H}_{\hat{Q}}\right]=-i \hat{H}_{\hat{Q}}, \quad\left[\hat{K}_{\hat{Q}}, \hat{P}_{\hat{Q}}\right]=i \hat{P}_{\hat{Q}}
$$

For this fact it is important that the boost operator $\hat{K}_{L C}$ and the projection operator on positive momentum states $\hat{Q}$ commute $\left(\left[\hat{K}_{L C}, \hat{Q}\right]=0\right)$. This is intuitively clear because of the simple form of the boost transformation. As a consequence, the decomposition of the Fock space into spaces of definite particle number and projection on positive momenta is a Lorentz invariant concept. This phenomenon is also valid in 1+1-dimensional QCD formulated in light-cone variables where it can be used for a valence quark approximation of mesons and baryons which is Lorentz-invariant [10].

\section{THE ANOMALY IN THE INFINITE MOMENTUM FRAME}

Weinberg motivated the use of light-cone variables by switching to a system which moves with infinite momentum [11]. We will show now that in this frame the effect of the anomaly

disappears. Consider the matrix element $\left\langle p_{1}^{\prime} p_{2}^{\prime}|\hat{Z}| p_{1} p_{2}\right\rangle$. After a straightforward, but lengthy calculation one finds in the infinite momentum limit $p_{1}, p_{2} \gg m$ and $p_{1}^{\prime}, p_{2}^{\prime} \gg m$

$$
\lim _{P \rightarrow \infty}\left\langle p_{1}^{\prime} p_{2}^{\prime}|\hat{Z}| p_{1} p_{2}\right\rangle=\frac{i m^{2} e^{2}}{4 \pi P^{4}} \delta\left(P-P^{\prime}\right)\left(\frac{1}{x^{2} y^{2}}+\frac{1}{(1-x)^{2}(1-y)^{2}}\right)
$$

where

$$
\begin{array}{lll}
p_{1}=x P & p_{2}=(1-x) P & P=p_{1}+p_{2} \\
p_{1}^{\prime}=y P & p_{2}^{\prime}=(1-y) P & P^{\prime}=p_{1}^{\prime}+p_{2}^{\prime} .
\end{array}
$$

The anomalous matrix element vanishes in the limit $P \rightarrow \infty$. Therefore it is possible to formulate relativistic quantum mechanics on a truncated Fock space in the infinite momentum frame. This is in accordance with Weinberg's motivation of the light cone.

The anomaly will only vanish if both particles carry a large momentum fraction, due to the singularities at $x=0, y=0$ and $x=1, y=1$. If quantum states with a non vanishing 
wave function at these singular points contribute in a relativistic wave equation, then the effect of antiparticles becomes important.

Moreover, it is not possible to boost wave functions or non scalar observables from the infinite momentum frame to another Lorentz frame, as we have no closed Poincaré algebra, which means we have no generator of unitary transformations $K$ in ordinary coordinates on a truncated Fock space. A two-body wave function in the infinite momentum frame is in general a complicated many-body wave function in the rest frame, containing a large number of particle-antiparticle pairs. Two problems arise if one tries to construct this wave function: first one has to find the boost generator in the presence of antiparticles, i.e. from the corresponding quantum field theory. This boost generator will in general be a dynamical quantity dependent on the interaction under consideration. The second problem is to calculate the boosted wave function $\psi^{\prime}$. It is given by the equation

$$
\left|\psi^{\prime}\right\rangle=\exp (i \lambda K)|\psi\rangle
$$

As $K$ is dynamical, i.e. it contains the interaction, this problem is as complicated as finding the full propagator of the quantum field theory. Therefore only scalar observables can be calculated in the infinite momentum frame or light-cone coordinates and compared with quantities in the rest frame of the two particles in ordinary coordinates.

\section{DISCUSSION}

We have seen that classical Poincaré symmetry of electrodynamics in one space dimension is lost if the theory is quantized on a truncated Fock space in ordinary coordinates. In a Hamiltonian formulation, this shows up as an anomaly in the Poincaré algebra. Almost all known examples of anomalies are restricted to relativistic quantum field theories. However, the loss of a classical symmetry after quantization is a more general phenomenon, which is related to the necessity of specifying a Hilbert space [12. This can be seen in the simple

case of the motion of a free particle in one dimension, with $H=p^{2}$ [13]. If one quantizes 
the theory on the Hilbert space $L_{2}[-\infty, \infty]$, the classical theory and the quantum theory are translationally invariant. The momentum operator $p$ generates a unitary transformation which translates the system according to

$$
\exp (i p \alpha) x \exp (-i p \alpha)=x+\alpha
$$

If the theory is quantized on a circle with periodic boundary conditions this symmetry breaks down to a discrete lattice symmetry, i.e. the parameter $\alpha$ has to be constrained to integral multiples of $2 \pi$ [13]. For a detailed discussion of a related problem in the presence of an external magnetic flux through a circle see [14]. Another example of an anomaly of a finite dimensional system which is closely related to the axial anomaly in QED, is the supersymmetric harmonic oscillator. The parity symmetry of the classical theory is broken by the ground state but all excited states appear in parity doublets [13.

The fact that the Poincaré anomaly is present in ordinary coordinates but not in lightcone coordinates indicates that a different Hilbert space is used in the two formulations. In light-cone coordinates the Fock space can be decomposed into spaces of a definite number of particles without violating Lorentz invariance, whereas in ordinary coordinates this is not possible. Therefore it is not possible to compare wave functions in the two formulations in a simple manner. As in the case of the infinite momentum frame, a two-body wave function in light-cone coordinates does not correspond to a two-body wave function in ordinary coordinates.

The discussion of the anomaly in the infinite momentum frame exemplifies that our results are consistent with the original motivation of light-cone coordinates [11]. From the structure of the anomalous matrix element in the infinite momentum frame, one can read off a necessary condition for the validity of the valence quark approximation in this Lorentz frame. If the momentum space wave functions do not vanish rapidly enough at the singular points $x=0$ and $y=0$, the anomaly is important also in this frame, therefore the truncation of Fock space violates Lorentz invariance.

Our results also clearly indicate that the so called "simplicity" of light-cone theories is 
rather deceptive and probably an artefact of working in 1+1-dimensions, as it relies on the exceptionally simple structure of the boost generator and the Fock space. Unfortunately, it is clear that two of the three boost generators will become dynamical quantities in $3+1$ dimensions also on the light-cone. As a result, a Lorentz invariant formulation of quantum mechanics will be as difficult as in ordinary coordinates. Therefore the intuition which is gained by studying lower dimensional theories may be misleading when phenomena in the real world, i.e. in 3+1-dimensions are to be understood. In addition, it will be much harder to implement e.g. rotational invariance on the light-cone, because the generators for this symmetry are also dynamical, in contrast to ordinary coordinates.

\section{ACKNOWLEDGEMENTS}

We would like to thank M. Burkardt, F. Lenz and D. Stoll for many fruitful discussions and M. Engelhardt and D. Kusnezov for reading the manuscript.

\section{APPENDIX A: CALCULATION OF MATRIX ELEMENTS OF $\hat{Z}$}

In this appendix we compute the matrix element $\left\langle x_{1}^{\prime} x_{2}^{\prime}\left|\hat{\Omega}_{1}\right| x_{1} x_{2}\right\rangle$ :

$$
\begin{aligned}
& \left\langle x_{1}^{\prime} x_{2}^{\prime}\left|\hat{\Omega}_{1}\right| x_{1} x_{2}\right\rangle= \\
& =\left\langle x_{1}^{\prime} x_{2}^{\prime}\left|\epsilon\left(\hat{x}_{1}-\hat{x}_{2}\right) \sqrt{\hat{p}_{1}^{2}+m^{2}}-\sqrt{\hat{p}_{1}^{2}+m^{2}} \epsilon\left(\hat{x}_{1}-\hat{x}_{2}\right)\right| x_{1} x_{2}\right\rangle= \\
& =\left\{\epsilon\left(x_{1}^{\prime}-x_{2}^{\prime}\right)-\epsilon\left(x_{1}-x_{2}\right)\right\} \int_{-\infty}^{\infty} d p_{1} \int_{-\infty}^{\infty} d p_{2}\left\langle x_{1}^{\prime} x_{2}^{\prime}\left|\sqrt{\hat{p}_{1}^{2}+m^{2}}\right| p_{1} p_{2}\right\rangle\left\langle p_{1} p_{2} \mid x_{1} x_{2}\right\rangle= \\
& =\left\{\epsilon\left(x_{1}^{\prime}-x_{2}^{\prime}\right)-\epsilon\left(x_{1}-x_{2}\right)\right\} \delta\left(x_{2}-x_{2}^{\prime}\right) \int_{-\infty}^{\infty} d p_{1} \sqrt{p_{1}^{2}+m^{2}} \frac{\exp \left(i p_{1}\left(x_{1}^{\prime}-x_{1}\right)\right)}{2 \pi} .
\end{aligned}
$$

For the integral on the right side one arrives at

$$
\begin{aligned}
& \int_{-\infty}^{\infty} \sqrt{p_{1}^{2}+m^{2}} \frac{\exp \left(i p_{1}\left(x_{1}^{\prime}-x_{1}\right)\right)}{2 \pi} d p_{1}= \\
& =2 \int_{0}^{\infty} \sqrt{p_{1}^{2}+m^{2}} \frac{\cos \left(p_{1} q\right)}{2 \pi} d p_{1}=\frac{2}{2 \pi} \int_{0}^{\infty}\left(\sqrt{p_{1}^{2}+m^{2}}-p_{1}+p_{1}\right) \cos \left(p_{1} q\right) d p_{1}
\end{aligned}
$$

where $q$ is defined as $q:=\left|x_{1}^{\prime}-x_{1}\right|$. Define 


$$
I_{1}:=\frac{2}{2 \pi} \int_{0}^{\infty} d p_{1} p_{1} \cos \left(p_{1} q\right)
$$

This integral has to be regularized

$$
I_{1, \text { reg }}:=\frac{2}{2 \pi} \lim _{\epsilon \rightarrow 0+} \operatorname{Re}\left\{\int_{0}^{\infty} d p_{1} p_{1} \exp \left(p_{1}(i q-\epsilon)\right)\right\}=\frac{-1}{\pi q^{2}} .
$$

For the integral $I_{2}$ defined as

$$
I_{2}:=\frac{2}{2 \pi} \int_{0}^{\infty} d p_{1}\left(\sqrt{p_{1}^{2}+m^{2}}-p_{1}\right) \cos \left(p_{1} q\right)
$$

one obtains after two partial integrations

$$
I_{2}=\frac{1}{\pi q^{2}}-\int_{0}^{\infty} d p_{1} \frac{m^{2} \cos \left(p_{1} q\right)}{\pi{\sqrt{p_{1}^{2}+m^{2}}}^{3} q^{2}} .
$$

Therefore one has finally [9]

$$
\begin{aligned}
& \int_{-\infty}^{\infty} d p_{1} \frac{\sqrt{p_{1}^{2}+m^{2}}}{2 \pi} \exp \left(i p_{1}\left(x_{1}^{\prime}-x_{1}\right)\right)= \\
& =I_{1}+I_{2}=-\int_{0}^{\infty} d p_{1} \frac{m^{2} \cos \left(p_{1} q\right)}{\pi{\sqrt{p_{1}^{2}+m^{2}}}^{3} q^{2}}=\frac{-m}{\pi\left|x_{1}^{\prime}-x_{1}\right|} K_{1}\left(m\left|x_{1}^{\prime}-x_{1}\right|\right)
\end{aligned}
$$

Hence

$$
\left\langle x_{1}^{\prime} x_{2}^{\prime}\left|\hat{\Omega}_{1}\right| x_{1} x_{2}\right\rangle=\left\{\epsilon\left(x_{1}^{\prime}-x_{2}^{\prime}\right)-\epsilon\left(x_{1}-x_{2}\right)\right\} \delta\left(x_{2}^{\prime}-x_{2}\right) \frac{-m}{\pi\left|x_{1}^{\prime}-x_{1}\right|} K_{1}\left(m\left|x_{1}^{\prime}-x_{1}\right|\right) .
$$

The calculation for $\left\langle x_{1}^{\prime} x_{2}^{\prime}\left|\hat{\Omega}_{2}\right| x_{1} x_{2}\right\rangle$ is completely analogous. From the two matrix elements (12) follows immediately.

\section{APPENDIX B: SOLUTION IN THE NONRELATIVISTIC LIMIT}

The nonrelativistic Hamiltonian for two particles interacting by a linear potential is

$$
\hat{H}=\sum_{i=1}^{2} \frac{\hat{p}_{i}^{2}}{2 m}+e^{2}\left|\hat{x}_{1}-\hat{x}_{2}\right| .
$$

After separating the center of momentum one has for the relative variable the following Schrödinger equation 


$$
-\frac{1}{m} \frac{\partial^{2}}{\partial r^{2}} \psi(r)+e^{2}|r| \psi(r)=E \psi(r)
$$

For $r>0$ one gets after the substitutions $y:=\left(e^{2} r-E\right) m$ and $z:=\left(e^{4} m^{2}\right)^{-\frac{1}{3}} y$

$$
\frac{d^{2} \psi(z)}{d z^{2}}-z \psi(z)=0
$$

The solution is 9

$$
\psi(z)=c_{1} \operatorname{Ai}(z)+c_{2} \operatorname{Bi}(z)
$$

$c_{2}$ must be zero because $\operatorname{Bi}(z)$ is not normalizable. For $r<0$ the procedure is similar. For the wave function one gets then

$$
\psi(r) \sim A i\left(m\left(e^{4} m^{2}\right)^{-\frac{1}{3}}\left(e^{2}|r|-E\right)\right)
$$

The energy eigenvalues are determined by the requirement that the wavefunction and its first derivative is continuous at the origin. Therefore one has

$$
A i^{\prime}\left(-m\left(e^{4} m^{2}\right)^{-\frac{1}{3}} E\right)=0
$$

and if the zeros of the first derivative of the Airy-function are denoted $a_{n}^{\prime}$ one has finally

$$
E_{n}=\left|a_{n}^{\prime}\right|\left(\frac{e^{4}}{m}\right)^{\frac{1}{3}}
$$

$\left|\psi_{n}(0)\right|^{2}$ is given by

$$
\left|\psi_{n}(0)\right|^{2}=\frac{1}{\left|a_{n}^{\prime}\right|}\left(\frac{e^{2} m}{8}\right)^{\frac{1}{3}}
$$

and therefore we have for $\left\langle\psi_{n}|\hat{Z}| \psi_{n}\right\rangle$

$$
\left\langle\psi_{n}|\hat{Z}| \psi_{n}\right\rangle=\int d r \psi(r)^{*} \frac{e^{2} i}{2 m^{3}} \delta(r) \psi(r)=\frac{i e^{2}}{2 m^{3}\left|a_{n}^{\prime}\right|}\left(\frac{e^{2} m}{8}\right)^{\frac{1}{3}} .
$$




\section{REFERENCES}

[1] H. Feshbach and F. Villars, Rev. Mod. Phys. 30 (1958) 24

[2] P. A. M. Dirac, Rev. Mod. Phys. 21 (1949) 392

[3] H. Goldstein, Classical Mechanics, Addison Wesley, Reading, Massachusetts, 1980

[4] J. D. Jackson, Classical Electrodynamics, Wiley, New York, 1962

[5] S. L. Adler, Phys. Rev. 177 (1969) 2426

[6] J. S. Bell and R. Jackiw, Nuovo Cim. A60 (1969) 47

[7] K. Fujikawa, Phys. Rev. D6 (1972) 477

[8] I. Bialynicki-Birula, Phys. Rev. D3 (1971) 864

[9] M. Abramovitz and I. Stegun, Handbook of Mathematical Functions, Dover Publications, New York, 1972, p. 375

[10] K. Hornbostel, S. J. Brodsky and H. C. Pauli, Phys. Rev. D41 (1990) 3814

[11] S. Weinberg, Physical Review 150 (1966) 1313

[12] L. O'Raifeartaigh amd A. Wipf, WKB Properties of the Time-Dependent Schrödinger System, Foundations of Physics Vol. 18, March 1988, pp. 307

L. O’Raifeartaigh, Axial Anomalies, preprint DIAS-STP-90-27, April 1990

[13] L. O'Raifeartaigh, Semiclassical Aspects of Quantum Field Theories, Lecture Notes of the Workshop "Topics in Field Theory", October 10-12 1994, GK-Notes University of Erlangen, unpublished

[14] N. S. Manton, Ann. Phys. 159 (1985) 220 


\section{FIGURES}

FIG. 1. Classical particle trajectory in Minkowski space.

FIG. 2. Quantum mechanical particle propagation in Minkowski space. 


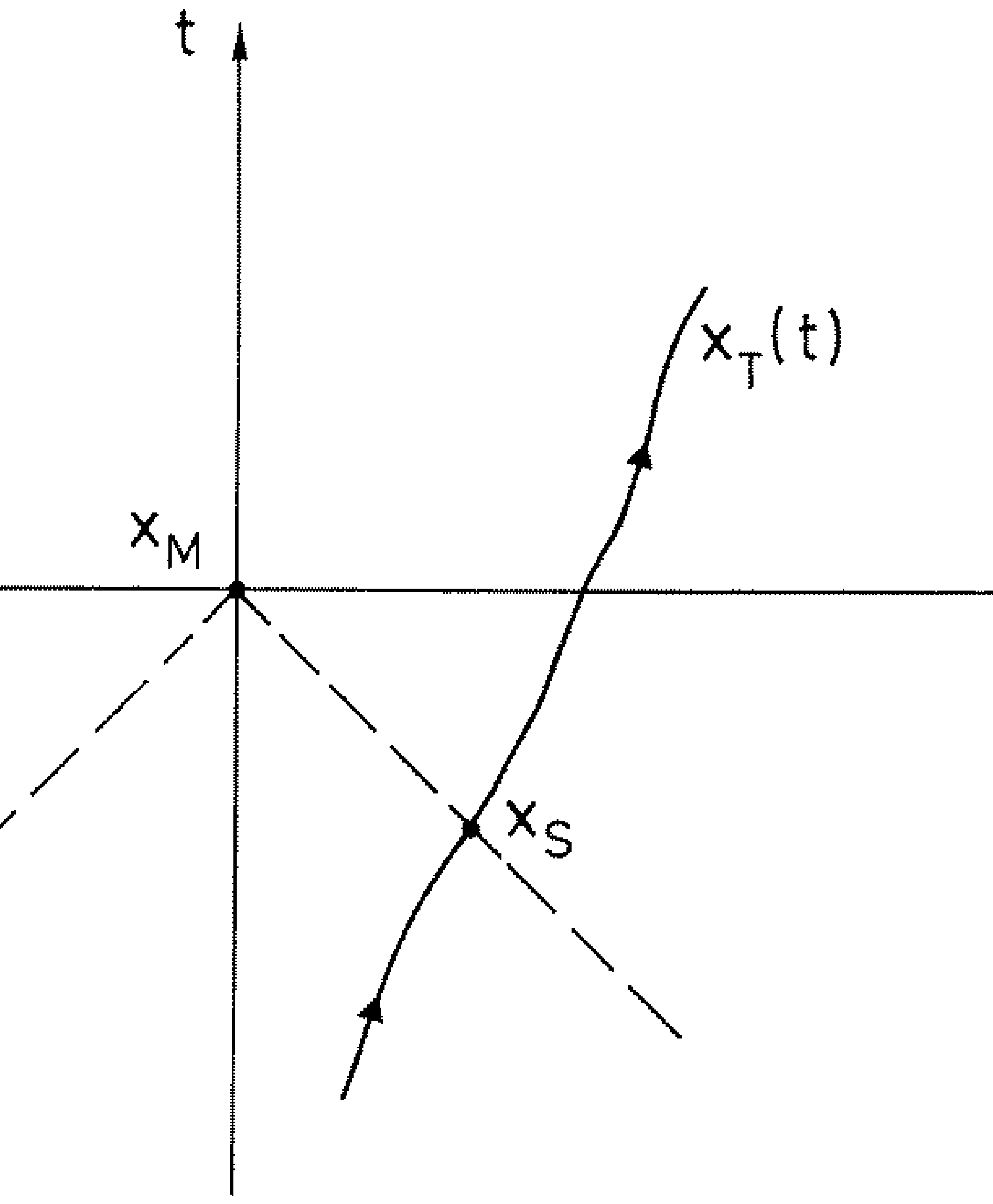


<smiles>[Y10]C([Y10])(C)CC</smiles> 